\title{
Desidratação e recomendações para a reposição hídrica em crianças fisicamente ativas
}

\author{
Dehydration and rehydration recommendations for physically active children
}

\author{
Luciana Rossi ${ }^{1}$, Vanessa Aparecida de B. Reis², Camila Ortis E. de Azevedo ${ }^{3}$
}

\section{RESUMO}

Objetivo: A prática de exercícios físicos, devido à produção inerente de calor, pode conduzir à desidratação. A maioria dos estudos que abordam os riscos da desidratação e fornecem recomendações de reposição hídrica é direcionada a indivíduos adultos residentes em regiões de clima temperado, porém, em regiões tropicais, pouco é conhecido sobre as necessidades de reposição hídrica em crianças fisicamente ativas. Esta revisão discute as recomendações para esta população e estabelece os riscos da prática esportiva em ambiente de clima tropical.

Fontes de dados: Análise sistemática com levantamento da literatura nacional (SciELO) e internacional (Medline) de artigos publicados entre 1972 e 2009, com os seguintes descritores isolados ou em combinação: hidratação, crianças, desidratação e reposição hídrica. Foram selecionados artigos publicados nas línguas portuguesa e inglesa.

Síntese dos dados: Observou-se que há riscos de desidratação e possível desenvolvimento de um quadro de hipertermia principalmente se as crianças são submetidas a condições climáticas desfavoráveis sem reposição hídrica adequada. O principal fator desencadeante da hipertermia é a menor adaptação das crianças aos extremos de temperatura, em comparação aos adultos, por possuírem área maior de superfície corporal e capacidade menor de termorregulação por evaporação.

Conclusões: Conhecidos os fatores intervenientes da desidratação, a melhor recomendação, perante uma con-

Instituição: Centro Universitário São Camilo, São Paulo, SP, Brasil ${ }^{1}$ Mestre em Ciência dos Alimentos pela Faculdade de Ciências Farmacêuticas da Universidade de São Paulo (USP); Coordenadora da Pós-graduação de Nutrição Esportiva e Estética do Centro Universitário São Camilo, São Paulo, SP, Brasil

${ }^{2}$ Especialista em Fisiologia e Metabolismo pelo Instituto de Ciências Biomédicas da USP, São Paulo, SP, Brasil

${ }^{3}$ Nutricionista, São Paulo, SP, Brasil dição climática sabidamente desfavorável, é estabelecer um plano impositivo de hidratação com bebida com sabor e acréscimo de carboidratos e sódio, evitando-se uma perda hídrica significativa, diminuição da performance e, principalmente, com o objetivo de reduzir os riscos à saúde impostos pela hipertermia e desidratação a crianças fisicamente ativas.

Palavras-chave: desidratação; exaustão por calor; sudorese; esforço físico.

\section{ABSTRACT}

Objective: The practice of physical exercises leads to dehydration due to heat production. Most studies that address the risks of dehydration and provide recommendations for restoring water are directed to adults living in temperate climate regions, but little is known about the needs of restoring water to physically active children in tropical regions. This review discusses the recommendations for this population and the risks of sports practice in tropical climate areas.

Data source: Systematic analysis of the national (SciELO) and international (Medline) literature from 1972 to 2009, with the following keywords, alone or in combination, in Portuguese and English: hydration, children, dehydration and water replacement.

Data synthesis: There are risks related to dehydration and possible development of hyperthermia especially in adverse
Endereço para correspondência:

Luciana Rossi

Rua Raul Pompéia, 144 - Pompéia

CEP 05025-010 - São Paulo/SP

E-mail: Irossi@scamilo.edu.br

Conflito de interesse: nada a declarar

Recebido em: 17/2/2009

Aprovado em: 6/8/2009 
weather conditions without adequate fluid replacement. The main trigger for hyperthermia is that, compared to adults, children are less able of adapting to extremes of temperature due to their higher body surface area and lower capacity of thermoregulation by evaporation. Studies on this subject are scarce in face of the questions still open.

Conclusions: Once dehydration factors are known, the best recommendation to aggressive climatic conditions is to establish a replacement program using flavored hydration beverage added with carbohydrates and sodium in order to avoid significant water losses and reduced performance, and to decrease health risks posed by hyperthermia and dehydration to physically active children.

Key-words: dehydration; heat exhaustion; sweating; physical exertion.

\section{Introdução}

A prática de exercícios e a adoção de uma dieta saudável desde a infância têm sido evidenciadas por inúmeros trabalhos como as principais estratégias para o combate à obesidade infantil. Encontram-se ainda relacionadas aos fatores modificáveis de risco: mudanças no estilo de vida como brincadeiras, tempo de televisão, computadores, insegurança para brincar na rua; e hábitos alimentares, por exemplo o apelo comercial dos produtos ricos em carboidratos simples, gordura e calorias e seu menor custo $^{(1)}$. Troncon et al ${ }^{(2)}$ revelam que, segundo a International Obesity Task Force (IOF), 22 milhões de crianças com menos de cinco anos têm sobrepeso. Esses altos índices de adiposidade estão diretamente relacionados ao desenvolvimento de diversas doenças crônicas na juventude que podem persistir na vida adulta, tais como hipertensão arterial, diabetes, dislipidemias, síndrome metabólica e doenças cardiovasculares ${ }^{(1,3)}$.

$\mathrm{Na}$ maioria dos países industrializados, os órgãos responsáveis pela saúde pública destacam a importância da participação de crianças em esportes ou exercícios regulares, sendo cada vez maior o número de crianças envolvidas em atividades que vão desde treinamento físicos até competição atlética ${ }^{(3-5)}$. A literatura a respeito da participação de crianças em atividade esportivas (prépúberes e púberes) tem sugerido que o período máximo de treinamento físico deve compreender 15 a 18 horas semanais para evitar que o crescimento seja comprometido $^{(4,6)}$. Porém, Georgopoulos et al ${ }^{(7)}$, em seu estudo com
255 ginastas femininas, encontrou a média semanal de prática de exercícios de 29,1 $\pm 15,3$ horas. Um dos riscos possíveis associados à prática de exercícios físicos extenuantes relaciona-se à consequente produção de calor. Para o bom desempenho das funções corporais, é importante que a temperatura seja mantida, com possibilidade de pequenas variações em torno de $37^{\circ} \mathrm{C}$, permitindo uma faixa de variação muito limitada $\left( \pm 1^{\circ} \mathrm{C}\right)$. Fora dessa amplitude, podem ocorrer consequências graves, tais como desidratação, deficiência na termorregulação corporal e redução do desempenho físico ${ }^{(8)}$. A reposição hídrica e de eletrólitos é importante na dieta de pessoas ativas em virtude da grande perda que pode ocorrer através do suor durante o exercício, particularmente em um ambiente quente e úmido ${ }^{(9)}$. Muitos estudos têm abordado os riscos e recomendações de reposição hídrica aplicada a indivíduos adultos fisicamente ativos, porém pouco é conhecido sobre os efeitos nocivos da desidratação e necessidades de reposição para crianças fisicamente ativas, mesmo levando-se em conta que os sistemas termorregulatórios das crianças são menos eficientes ${ }^{(3,10)}$.

O Brasil, país localizado em região tropical, é caracterizado por apresentar grandes variações climáticas devido a suas proporções continentais, com associação de altas temperaturas e umidade relativa na maior parte do ano. Estudos demonstram que jovens que participam de programas esportivos em regiões de clima tropical podem desenvolver um estado crônico de desidratação se não houver reposição hídrica adequada ${ }^{(11)}$. Prevenir tal situação em crianças é tão essencial quanto em adultos ${ }^{(10)}$.

Mediante os riscos impostos pela hipertermia e desidratação em crianças e adolescentes, esta revisão tem como objetivo discutir as recomendações de hidratação frente às necessidades impostas pela prática esportiva e acrescentar elementos científicos à discussão de recomendações para aplicação de estratégias de reidratação para países situados em regiões de clima tropical, como o Brasil.

\section{Fontes de dados}

Realizou-se uma revisão sistemática de artigos científicos com o objetivo de avaliar os efeitos da desidratação em crianças fisicamente ativas. Como fatores de inclusão foram selecionados artigos publicados em revistas indexadas entre 1972 e 2009, utilizando os bancos de dados eletrônicos Scientific Eletronic Library (SciELO Brasil) e National Library of Medicine (Medline, Estados Unidos) com os seguintes 
descritores, isoladamente ou em combinação: hidratação, crianças, desidratação e reposição hídrica (em inglês: dehydration, hydration, children, sport, hypohydration, drinking behavior, thermoregulation). Como critério de inclusão dos artigos, analisou-se a procedência da revista e seu fator de impacto, além do desenho de estudo, preferindo-se os clínicos. Adicionalmente, a pesquisadora principal selecionou os artigos de acordo com o grupo de pesquisa, o desenho experimental com presença ou não de grupo controle, o tamanho da amostra, a faixa etária e a análise estatística empregada. Também foram feitas pesquisas em livros.

\section{Resultados}

\section{Termorregulação e hipertermia em crianças}

A temperatura corporal está em constante equilíbrio, mantido pela integração de mecanismos que alteram a transferência de calor da região central para a periferia do corpo (Figura 1). A temperatura periférica pode variar de acordo com a do ambiente, mas a central deve-se manter em $37 \pm 1^{\circ} \mathrm{C}$. Se o ganho de calor ultrapassar sua perda, processo que ocorre comumente na prática de atividades vigorosas em ambiente quente, a temperatura central eleva-se e o calor produzido é conduzido pela circulação sanguínea, sendo então transferido para a pele a fim de ser dissipado para o ambiente; por outro lado, em ambiente frio, a perda de calor é maior que a sua produção, fazendo com que a temperatura corporal central diminua ${ }^{(12)}$.

Com a exposição do atleta ao estresse térmico durante o exercício, diversas situações podem ocorrer, incluindo elevação da temperatura cutânea, vasodilatação e aumento da transpiração ${ }^{(13)}$. Em geral, estas ocorrências facilitam a transferência efetiva de calor para o ambiente, o que minimiza a elevação da temperatura central do corpo, processo denominado termorregulação. Esta, ainda é afetada pelo nível de atividade, estado de aclimatização e intensidade relativa de trabalho ${ }^{(14)}$; mas, quando realizado em condições extremas, o exercício prolongado pode resultar em distúrbios térmicos com significado clínico $^{(15)}$. A capacidade de troca de calor do organismo com o ambiente depende diretamente de quatro mecanismos específicos: condução, radiação, evaporação e convecção ${ }^{(12)}$. As crianças têm uma área maior de superfície corporal que os adultos, o que facilita a transferência de calor por convecção e por radiação e possivelmente as coloca em desvantagem quando expostas ao frio ${ }^{(16)}$. Entretanto, a perda de calor por evaporação em crianças é menos eficiente ${ }^{(17,18)}$. Em climas

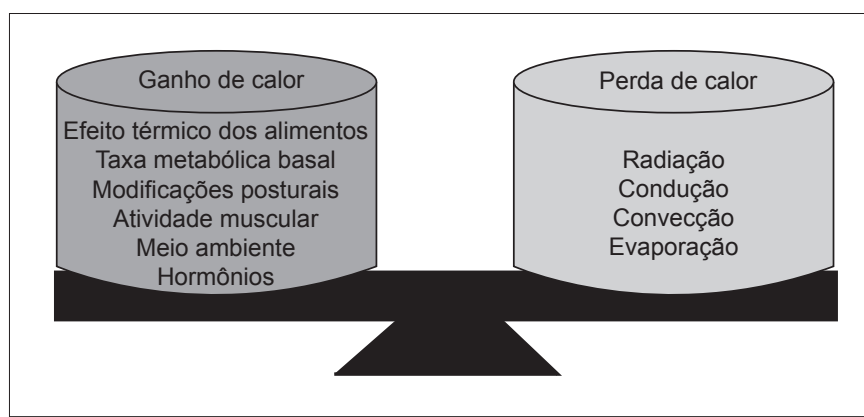

Figura 1 - Fatores que contribuem com o ganho e a perda de calor.

muito quentes, quando a temperatura ambiente supera em $10^{\circ} \mathrm{C}$ a temperatura da superfície corporal, a tolerância ao calor entre crianças é bastante reduzida, acompanhada pela elevação na temperatura central e armazenamento de calor, bem como uma baixa taxa de transpiração, podendo ser 2,5 vezes menor que a dos adultos expostos às mesmas condições climáticas ${ }^{(9,14)}$. Os indivíduos incapazes de se adaptarem fisiologicamente a ambientes quentes podem apresentar uma síndrome denominada intolerância ao calor, que é comumente descrita em crianças praticantes de atividade física ${ }^{(19)}$. Tal intolerância se traduz em sintomas de desidratação, como sede, perda de apetite e fadiga, entre outros ${ }^{(20)}$. Alguns fatores explicariam a menor adaptação das crianças aos extremos de temperatura em relação aos adultos ${ }^{(21,22)}$, dentre eles: uma área maior de superfície corporal, com um ganho de calor maior em dias quentes e perda de calor em dias frios; produção maior de calor metabólico por unidade de massa durante a atividade física, incluindo caminhadas e corridas, e a capacidade consideravelmente menor de sudorese, com redução da habilidade de dissipar o calor corporal através da evaporação ${ }^{(11,23,24)}$.

\section{Desidratação e taxa de sudorese}

O esforço físico associado ao estresse térmico aumenta a produção do suor e o fluxo sanguíneo cutâneo para combater o aumento crescente da temperatura. O processo de evaporação induz ao resfriamento da superfície corporal e do sangue, dissipando o calor interno. A transpiração aumentada durante o exercício, em temperaturas elevadas, conduz rapidamente à desidratação ${ }^{(25)}$. Como grande parte da água perdida é proveniente do fluido extracelular que inclui o plasma sanguíneo, ocorre diminuição deste volume e da capacidade de trabalho, já que este precisa mantido para suprir o oxigênio e os substratos para os músculos e 
dissipar o calor pela superfície da pele ${ }^{(26)}$. A capacidade de sudorese das crianças é baixa e, além do risco de hipertermia, a desidratação está fortemente associada à fadiga ${ }^{(27)}$. Bar-Or $e t$ a $l^{(10)}$ observaram que há um aumento de quase $0,3^{\circ} \mathrm{C}$ na temperatura central de crianças entre dez e 12 anos de idade para cada $1 \%$ de peso corporal perdido, sendo que, em adultos, a temperatura central é elevada em $0,1-0,2^{\circ} \mathrm{C}$ para a mesma perda percentual. Segundo a $\mathrm{ACSM}^{(13)}$, há mais de um método para avaliar o estado de hidratação e, na declaração de evidência, pondera como Evidência de categoria A, ou seja, recomendação baseada em evidência experimental consistente e de boa qualidade, que as mudanças no peso corporal refletem as perdas pela sudorese durante o exercício e podem ser utilizadas para calcular as necessidades individuais de reposição hídrica para atividades físicas específicas e condições ambientais. Sendo assim, a diferença percentual de peso pode ser utilizada como um dos parâmetros de avaliação do grau de desidratação. Em adultos, a perda corporal hídrica entre 1 e $2 \%$ predispõe a um aumento considerável na temperatura corporal; com aproximadamente $3 \%$ de desidratação há redução no desempenho; com 4 a $6 \%$ pode ocorrer fadiga térmica e, a partir de $6 \%$, há risco de choque térmico e, progressivamente, coma e morte ${ }^{(25)}$. Em crianças, uma redução em torno de $1 \%$ do peso corporal induzida pela sudorese reduz significativamente a habilidade de realização de exercício físico ${ }^{(27)}$. Por razões éticas, os graus de desidratação em experimentos feitos com crianças não devem exceder 2 a $3 \%$ de perda de peso corporal $^{(3,10)}$. Com a progressão do processo de desidratação, ocorre uma combinação de sensações indesejáveis incluindo boca, garganta e lábios secos e rachados; cansaço, irritabilidade, dor de cabeça, perda do apetite e desconforto gástrico ${ }^{(28)}$. Como consequência, o mecanismo da sede em adultos é estimulado quando o peso corporal diminui de 0,5 a $1,0 \%$, sendo resultado de uma interação complexa do sistema fisiológico e do ambiente que leva à desidratação. Assim, a sede é estimulada por dois mecanismos homeostáticos principais: aumento da tonicidade celular (desidratação celular), detectada pelos osmorreceptores do sistema nervoso central, e pela redução do volume extracelular (desidratação extracelular), monitorada pelos barorreceptores (Figura 2). A relação da influência da desidratação intra e extracelular ainda é incerta em crianças, restando muitas questões a respeito das diferenças na sensação de sede e no consumo de líquidos ${ }^{(29)}$.

A desidratação extracelular é a mais complexa, com a participação de um duplo feedback, envolvendo barorreceptores ativados pela diminuição no volume sanguíneo. Há também a ativação do sistema renina-angiotensina-aldosterona em

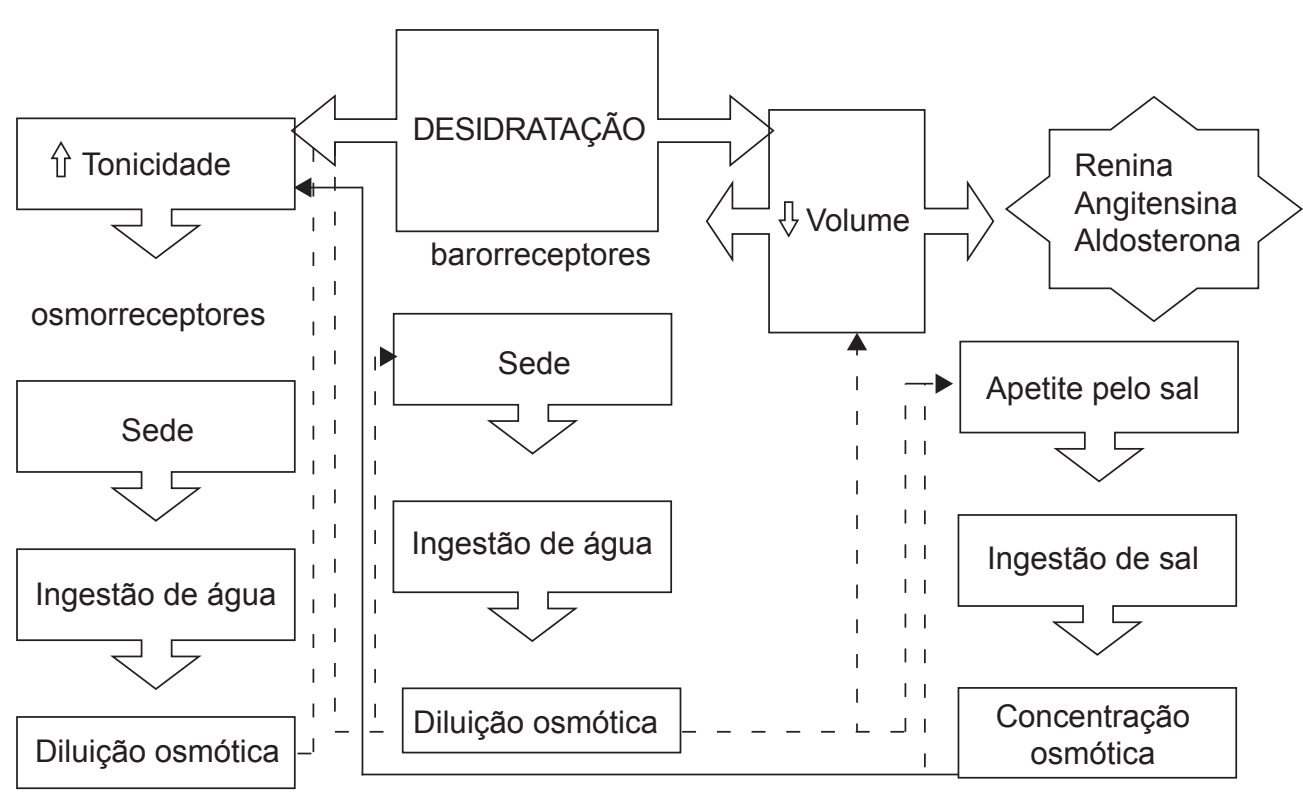

Feedback negativo

Feedback positivo

Fonte: adaptado de Kenney e Chiu(29).

Figura 2 - Modelo simplificado para o controle homeostático da sede e mecanismos de controle da desidratação intra e extracelular. 
resposta à perfusão renal diminuída. Além dos controles homeostáticos da hidratação, há vários controles nãohomeostáticos de estímulo à sede (Figura 3). Tais estímulos incluem uma ampla influência psicológica e ambiental, tais como disponibilidade da bebida, gosto, temperatura, associação com refeições e hábitos sociais ${ }^{(28)}$. O balanço hídrico corporal necessita de uma integração constante entre a ativação dos osmorreceptores hipotalâmicos e dos barorreceptores vasculares, de modo que a ingestão de líquidos seja similar à perda, e esta ocorre mais rapidamente durante o exercício do que o repouso devido à constante taxa de transpiração ${ }^{(15)}$.

Comparados aos outros mamíferos, os humanos apresentam uma taxa de ingestão voluntária de líquidos durante ou depois da perda muito mais lenta. Este atraso na reidratação é tecnicamente chamado de desidratação voluntária ${ }^{(30)}$, cuja causa encontra-se no atraso ou ingestão insuficiente de líquidos para compensar a perda durante a atividade física ${ }^{(31)}$. As crianças, assim como os adultos, não ingerem voluntariamente a quantidade necessária para repor as perdas pelo suor, o que pode causar desidratação durante e até mesmo antes dos exercícios ${ }^{(8)}$.

Durante o exercício, a ingestão hídrica em crianças dependerá da sua taxa de sudorese para a modalidade esportiva ${ }^{(23)}$, e esta já foi determinada para meninos (11 a 14 anos) para as modalidades de tênis, artes marciais, luta greco-romana, boxe, campo e trilha, natação em $556 \pm 110 \mathrm{~mL} / \mathrm{h}$ e corrida, ciclismo entre outros, em meninos (13 \pm 1 anos) de $937 \pm 342 \mathrm{~mL} / \mathrm{h}$ e meninas $(17 \pm 1$ anos) de $567 \pm 110 \mathrm{~mL} / \mathrm{h}^{(27)}$. Estudos laboratorialmente controlados com a modalidade de duatlo observaram que crianças mais jovens do sexo masculino (12,5-14,8 anos) exibiram taxa de sudorese $(\mathrm{mL} / \mathrm{h})$ menor do que as mais velhas $(15,0-17,1$ anos), ou seja: $640 \pm 370$ versus $1260 \pm 170 \mathrm{~mL} / \mathrm{h}$. O mesmo foi observado entre as crianças do sexo feminino para equivalentes faixas etárias: $510 \pm 26$ versus $710 \pm 379 \mathrm{~mL} / \mathrm{h}^{(27)}$. Esses resultados indicam globalmente a relação entre idade e gênero como possíveis determinantes da taxa de sudorese, podendo haver também diferenças nessa taxa em crianças fisicamente ativas de acordo com a posição de jogo, como no caso o futebol ${ }^{(32)}$.

\section{Recomendações de hidratação em crianças fisicamente ativas}

As recomendações de consumo hídrico para crianças são fornecidas pelo Institute of Medicine ${ }^{(33)}$ pelos valores de AI (Adequate Intake), estabelecidos com base em estimativas da ingestão assumidas como adequadas para um grupo de indivíduos, observadas ou determinadas experimentalmente (Tabela 1) ${ }^{(34)}$. Porém, tais recomendações provavelmente não são aplicáveis a crianças fisicamente ativas, principalmente durante o exercício prolongado, pois há perda de quantidade considerável de eletrólitos e água pelo suor, e caso a atividade física seja prolongada ( $>1$ hora) ou intensa e intermitente, sódio e carboidratos devem ser adicionados a uma solução preferencialmente flavorizada ${ }^{(23)}$. Duas razões são destacadas

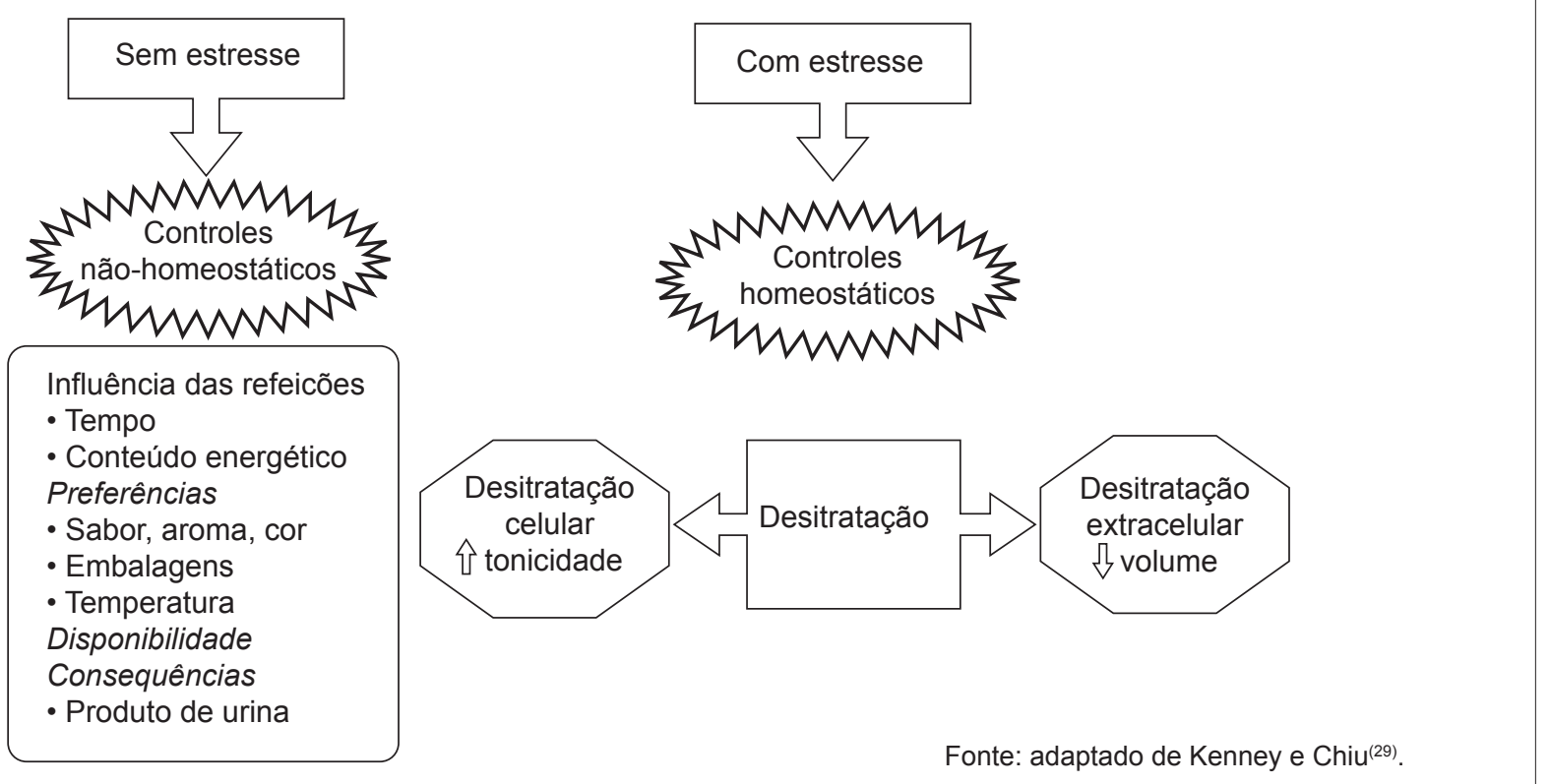

Figura 3 - Controles homeostáticos e não-homeostáticos da sede em humanos. 
Tabela 1 - Valores de Al (Adequate Intake)* para reposição hídrica, segundo o sexo

\begin{tabular}{lc}
\hline & Al (L/dia) \\
\hline Masculino & \\
1 a 3 anos & 1,3 \\
4 a 8 anos & 1,7 \\
9 a 13 anos & 2,4 \\
14 a 18 anos & 3,3 \\
Feminino & \\
1 a 3 anos & 1,3 \\
4 a 8 anos & 1,7 \\
9 a 13 anos & 2,1 \\
14 a 18 anos & 2,3 \\
\hline
\end{tabular}

*Representa a necessidade de água total para clima temperado. Todas as fontes de água podem contribuir para a necessidade total de água (chás, cafés, sucos, água e umidade dos alimentos). Fonte: $\mathrm{IOM}^{(33)}$.

para o estímulo à ingestão de líquidos durante atividade física por crianças: minimizar o efeito da desidratação e hipertermia e, deste modo, aumentar a efetividade e segurança do treinamento; e a importância de se testarem diferentes bebidas para a reposição para saber qual a mais eficaz para a reidratação de cada atleta ${ }^{(35)}$.

\section{Composição da bebida reidratante}

Meyer et al $^{(36)}$ verificaram que a percepção de sede em crianças não foi afetada pela concentração de sódio da bebida ingerida. Isto é particularmente importante para o entendimento de suas respostas frente aos diferentes tipos de estratégias de hidratação e otimização da reposição de líquidos. A concentração de sódio e cloreto plasmáticos sofre alterações com o aumento da idade, o que também diferencia a concentração encontrada no suor. As crianças têm uma concentração de sódio no suor de aproximadamente $40 \mathrm{mEq} / \mathrm{L}$ e os adultos, de $60 \mathrm{mEq} / \mathrm{L}^{(37)}$. As concentrações de sódio e cloreto perdidos pela transpiração em pré-púberes são menores do que em púberes e adultos de ambos os gêneros, entretanto, a concentração de potássio é maior em crianças $^{(10,14)}$. A quantidade baixa de sódio perdida durante a transpiração sugere que o déficit na concentração de sódio em crianças pode ser menor do que em adultos, e implicaria baixo risco de hiponatremia ${ }^{(38)}$.

Para estimular a ingestão de líquidos pelas crianças, pode-se oferecer bebidas coloridas (como suco de frutas), usar recipientes atraentes, transformar o suco em picolé, evitando os corantes artificiais, congelar sucos em cubos de gelo, entre outras estratégias ${ }^{(35)}$.

Diversos estudos com adultos têm indicado a importância do sabor e da temperatura da bebida a ser ingerida para estimular o consumo voluntário. Wilk et al ${ }^{(39)}$, analisando garotos entre dez e 12 anos, observaram que a ingestão de bebida ad libitum sem sabor resultou em desidratação progressiva durante um período de três horas de exercício intermitente a $35^{\circ} \mathrm{C}$ e 45 a $50 \%$ de umidade relativa do ar (URA). Relataram também que o aumento de ingestão voluntária da bebida com sabor (flavorizada) acrescida de carboidratos e de cloreto de sódio, em comparação à ingestão de água, pode ter sido fisiológica ou comportamental. O mecanismo fisiológico mais provável é que o cloreto de sódio adicionado ao repositor tenha influenciado no aumento da osmolalidade do líquido corporal. Isto, por sua vez, pode ter fornecido um estímulo maior aos osmorreceptores hipotalâmicos, que desencadearam a sensação de sede. Wilk e Bar-Or ${ }^{(40)}$ analisaram, em crianças (nove a 12 anos), a relação de bebidas flavorizadas e a ingestão voluntária. Em condições climáticas de $35^{\circ} \mathrm{C}$ e $50 \%$ de URA, foi oferecida água ou bebida aromatizada sabor uva ou bebida sabor uva com $6 \%$ de carboidratos e $18 \mathrm{mmol} / \mathrm{L}$ de cloreto de sódio. A ingestão total, ao longo de três horas de estudo, foi maior para a bebida flavorizada com carboidratos e eletrólitos $(1,157 \mathrm{~L})$ e menor para a água $(610 \mathrm{~mL})$. A desidratação foi mais expressiva com a ingestão de água, seguida pela de bebida aromatizada. Nesse estudo, também constatou-se uma hiperidratação quando foi ingerida a bebida flavorizada acrescida de carboidrato e eletrólitos, com aumento médio de $0,47 \%$ do peso inicial. Já Meyer $e t a l^{(39)}$ administraram quatro tipos diferentes de bebidas em crianças que treinaram com temperatura de $35^{\circ} \mathrm{C}$ e $45 \%$ URA para saber a melhor composição para esta faixa etária. Ofereceu-se água ou solução contendo $6 \%$ de carboidratos ou com $6 \%$ de carboidratos e $8,8 \mathrm{mmol} / \mathrm{L}$ de sódio ou ainda a mesma concentração de carboidratos com 18,5 mmol/L de sódio. Em todos os grupos, não houve diferença entre o aumento da temperatura corporal, a taxa cardíaca e o tempo de exaustão.

Rivera-Brown et al ${ }^{(8)}$ estudaram os níveis de hidratação voluntária em crianças residentes de uma região de clima tropical, com temperatura ambiente de $30^{\circ} \mathrm{C}$. Foram ofertadas água e água flavorizada acrescida de $6 \%$ de carboidratos e $18 \mathrm{mmol} / \mathrm{L}$ de sódio. Observou-se maior ingestão hídrica com a bebida carbonatada, sendo mantido o estado de hidratação normal em relação ao consumo de água ${ }^{(41)}$.

Quanto à preferência pelo sabor da bebida durante a atividade física, um estudo com crianças (nove a 13 anos) de ambos os sexos registrou maior preferência por uva em relação a laranja e maçã $\tilde{a}^{(3,36)}$. 


\section{Recomendações adicionais}

A temática científica envolvendo a composição ideal de uma bebida reidratante a ser utilizada durante a prática esportiva e os fatores intervenientes, como duração e intensidade do exercício, quantidade de suor, perdas de eletrólito, palatabilidade da bebida, além de idade e grau de aclimatação ao calor, é um campo extremamente profícuo nas áreas de saúde e rendimento esportivo. Grande parte das pesquisas a respeito da reidratação frente a uma desidratação provocada pelo exercício é realizada em adultos, sendo algumas das recomendações aplicadas para crianças obtidas a partir desses dados. Entretanto, esta população necessita de recomendações específicas devido às diferentes características fisiológicas, como taxa de transpiração, área de superfície corporal, perda de eletrólitos pelo suor ${ }^{(42)}$ entre outros fatores. A maioria dos estudos conduzidos com crianças foi realizada em países de clima temperado, com tentativa de reproduzir em ambiente laboratorial as condições climáticas adversas comumente encontradas em países tropicais, caracterizadas por altas temperaturas e elevada umidade relativa do ar. Porém, reproduzir laboratorialmente essas condições climáticas durante a atividade física é diferente da realidade de ser exposto constantemente a este ambiente, podendo o indivíduo adquirir mais facilmente um estado crônico de desidratação, com consequente estresse devido ao aumento da temperatura corporal ou ineficiência dos processos de termorregulação inerentes à idade. É importante enfatizar que a umidade do ar é o maior componente do estresse térmico e, na maioria das vezes, mais importante do que a própria temperatura ambiente ${ }^{(21)}$. As crianças e adolescentes que se exercitam no calor devem ser monitorados cuidadosamente em busca de sinais de estresse térmico, principalmente aquelas que possuem maior adiposidade pelo fato de apresentarem menor capacidade de dissipar o calor corporal ${ }^{(11)}$.

$\mathrm{A} \mathrm{AAP}^{(11,28)}$ recomenda que, para crianças e adolescentes em regiões de clima quente e úmido, que são particularmente mais agressivas para a prática esportiva dessa população, quando comparadas a locais de clima temperado ${ }^{(43,44)}$ as seguintes orientações devam ser observadas:

1. A intensidade das atividades $>15$ minutos deve ser reduzida sempre que a umidade relativa do ar, radiação solar e temperatura estiverem em níveis críticos (Tabela 2), ou zonas de perigo (zona 3) (Figura 4). Uma alternativa para aumentar o período de descanso em condições adversas é realizar maior número de substituições dos participantes da competição ou treinamento.

2. No início de programas de exercícios extenuantes ou após viajar para um local de clima mais quente, a intensidade e duração do exercício deve ser inicialmente limitada e gradualmente aumentada ao longo de um período de dez a 14 dias, permitindo a aclimatação. Caso não haja disponibilidade, tanto a prática como a competição devem ser reduzidas.

3. Antes de atividade física prolongada, as crianças devem estar bem hidratadas. Durante a atividade, ingerir líquidos periodicamente (ex: $150 \mathrm{~mL}$ de água potável fria ou solução flavorizada e salgada a cada 20 minutos para crianças pesando até $40 \mathrm{~kg}$ e $250 \mathrm{~mL}$ para adolescentes pesando $60 \mathrm{~kg}$ ), mesmo na ausência de sede. A pesagem antes e após a sessão de treinamento, preferencialmente vestindo roupas leves ou sem elas, pode avaliar o estado de hidratação.

4. Roupas e uniformes devem ser de cor clara, leves e limitados a uma camada de material absorvente para facilitar a evaporação do suor. Peças de roupas úmidas devem ser repostas por secas. Roupas de borracha nunca devem ser utilizadas para perda de peso.

5. O uso de protetor solar, roupas leves e chapéu é recomendado sempre que possível. Os supervisores devem ser cuidadosos em relação à duração e à intensidade do exercício realizado sob o calor.

As crianças devem ser conscientizadas e alertadas sobre a importância da ingestão de líquidos durante o exercício a

Tabela 2 - Restrições de atividades em diferentes níveis para a prevenção do estresse térmico em crianças*

\begin{tabular}{ll}
\hline WBGT $\left({ }^{\circ} \mathbf{C}\right)$ & Restrições de atividades \\
\hline$<24,0$ & $\begin{array}{l}\text { Todas as atividades são permitidas, mas é preciso estar alerta aos sintomas relacionados à lesão } \\
\text { térmica em exercícios prolongados }\end{array}$ \\
24,0 a 25,9 & Períodos de descanso mais longos devem ser promovidos; estimular bebidas a cada 15 minutos \\
26,0 a 29,0 & Parar a atividade de crianças aclimatizadas e com risco elevado \\
$>29,0$ & Cancelar todas as atividades atléticas
\end{tabular}

*WBGT (Wet Bulb Globe Temperature) não é a temperatura do ar e sim um índice de estresse térmico ambiental que depende da temperatura ambiente, da umidade relativa e do calor radiante. Pode ser mensurado em situações de campo pelo uso de três termômetros comercialmente disponíveis. Fonte: adaptado de $\mathrm{AAP}^{(21)}$. 


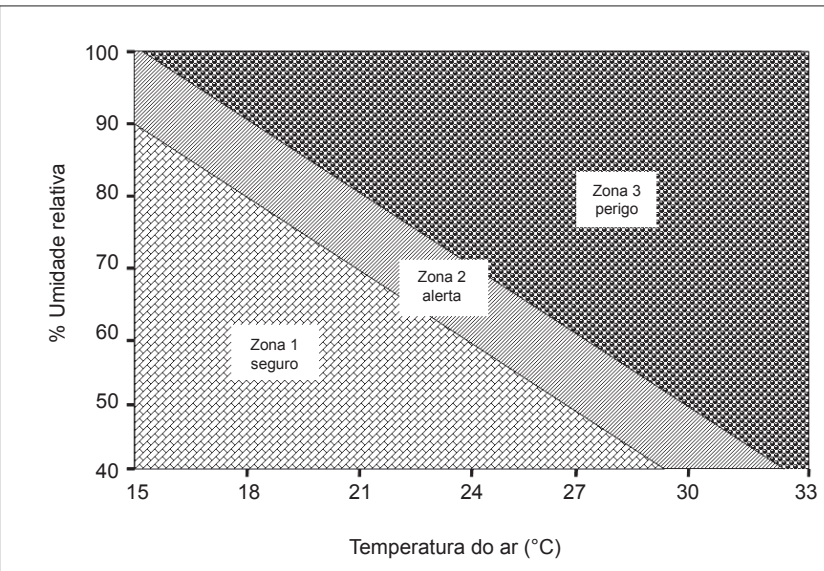

Fonte: modificado de APA ${ }^{(11)}$.

Figura 4 - Guia climático para prevenção de hipertermia em crianças fisicamente ativas durante o exercício prolongado.

fim de reduzir os riscos à saúde impostos pela hipertermia, além de tal conduta aumentar seu rendimento esportivo.

\section{Conclusão}

Há um número crescente de crianças inseridas no contexto da prática da atividade física tanto para lazer e competição como para prevenir a obesidade, em idade cada vez mais precoce. Contrariamente a este crescimento, é pequeno o número de estudos que investigam as atividades esportivas envolvendo crianças e as recomendações adequadas de hidratação, que são na sua maioria derivadas de estudos com população adulta. Considerando-se que as crianças são um grupo de risco para a desidratação, aquelas fisicamente ativas merecem uma atenção especial, pois, em relação aos adultos, possuem mecanismo de estímulo da sede menos eficiente, subestimando suas necessidades hídricas e acarretando maior desidratação voluntária. Diversos estudos com amostras de crianças e adolescentes demonstram que a reidratação é mais eficiente com a oferta de bebidas flavorizadas, atrativas (coloridas), geladas e com o acréscimo de carboidrato a $6 \%$, além de eletrólitos, embora não haja consenso sobre a adequada formulação destas soluções. Conclui-se, finalmente, que a melhor recomendação para este grupo, perante a condição climática de nosso país, seja estabelecer um plano de hidratação que não permita um quadro de perda hídrica significativa, com monitoramento da variação de peso antes e após a sessão de exercício a fim de manter a performance durante a atividade e, principalmente, não colocar a criança em risco pelos efeitos induzidos pela hipertermia.

\section{Referências bibliográficas}

1. Mello ED, Luft VC, Meyer F. Childhood obesity - Towards effectiveness. J Pediatr (Rio J) 2004;80:173-82.

2. Troncon JK, Gomes JP, Guerra Jr G, Lalli CA. Obesity prevalence among students of a public school and a Pediatric out-patient clinic of a university hospital. Rev Paul Pediatr 2007;25:305-10

3. Juswiak CR, Paschoal VC, Lopez FA. Nutrição e atividade física. J Pediatria (Rio J) 2000;76 (Supl 3):S349-58.

4. Alves C, Lima RV. Linear growth and puberty in children and adolescents: effects of physical activity and sports. Rev Paul Pediatr 2008;26:383-91.

5. Cotugna N, Vickery CE, McBee S. Sports nutrition for young athletes. J Sch Nurs 2005;21:323-8.

6. Silva CC, Goldberg TB, Teixeira AS, Marques I. O exercício físico potencializa ou compromete o crescimento longitudinal de crianças e adolescentes? Mito ou verdade? Rev Bras Med Esporte 2004;10:520-4.

7. Georgopoulos N, Markou K, Theodoropoulou A, Paraskevopoulou P, Varaki L, Kazantzi Z et al. Growth and pubertal development in elite female rhythmic gymnasts. J Clin Endocrinol Metab 1999;84:4525-30.

8. Rivera-Brown AM, Gutiérrez R, Gutiérrez JC, Frontera WR, Bar-Or O. Drink composition, voluntary drinking, and fluid balance in exercising, trained, heatacclimatized boys. J Appl Physiol 1999;86:78-84.

9. Bar-Or O, Dotan R, Inbar O, Rotshtein A, Zonder H. Voluntary hypohydration in 10- to 12-year-old boys. J Appl Physiol 1980;48:104-8.

10. Bar-Or O, Wilk B. Water and electrolyte replenishment in the exercising child. Int J Sport Nutr 1996;6:93-9.

11. American Academy of Pediatrics Committee on Sports Medicine: Climatic heat stress and the exercising child. Pediatrics 1982;69:808-9.

12. Guerra I, Gomes MR, Tirapegui J. Hidratação no esporte. In: Tirapegui J.

Nutrição, metabolismo e suplementação na atividade física. São Paulo: Atheneu; 2005. p.107-18.

13. Sawka MN, Burke LM, Eichner R, Maughan RJ, Montain SJ, Stachenfeld NS Exercise and fluid replacement. Med Sci Sports Exerc 2007;28:377-90.

14. Falk B, Bar-Or O, Calvert R, MacDougall JD. Sweat gland response to exercise in the heat among pre-, mid- and late-pubertal boys. Med Sci Sports Exerc 1992;24:313-9.

15. Murray R. Fluid needs in hot and cold environments. Int $\mathrm{J}$ Sport Nutr 1995;5:S62-S73.

16. Smolander J, Bar-Or O, Korhonen O, Ilmarinen J. Thermoregulation during rest and exercise in the cold in pre- and early pubescent boys and in young men. J Appl Physiol 1992;72:1589-94.

17. Hebestreit H, Bar-Or O, Mckinty C, Riddell M, Zehr P. Climate-related corrections for improved estimation of energy expenditure from heart rate in children. J Appl Physiol 1995;79:47-57.

18. Falk B, Bar-Or O, MacDougall D. Thermoregulatory responses of premid-, and late-pubertal boys to exercise in dry heat. Med Sci Sports Exerc 1992;24:688-94.

19. Epstein Y, Shapiro Y, Brill S. Role of surface area-to-mass ratio and work efficiency in heat intolerance. J Appl Physiol 1983;54:831-6.

20. Carvalho T, Rodrigues T, Meyer F, Lancha Jr AH, De Rose EH, Nóbrega AC et al. Modificações dietéticas, reposição hídrica, suplementos alimentares e drogas: comprovação de ação ergogênica e potenciais riscos para a saúde. Rev Bras Med Esporte 2003;9:43-56.

21. AAP (American Academy of Pediatrics). Climatic heat stress and the exercising child and adolescent. Pediatrics 2000;106:158-9.

22. Inbar O, Morris N, Epstein Y, Gass G. Comparison of thermoregulatory 
responses to exercise in dry heat among prepubertal boys, young adults and older males. Exp Physiol 2004;89:691-700.

23. Meyer F, O'Connor H, Shirreffs SM. Nutrition for the young athlete. J Sports Sci 2007;25:S73-82.

24. Machado-Moreira CA, Vimieiro-Gomes AC, Silami-Garcia E, Rodrigues LO. Hidratação durante o exercício: a sede é suficiente? Rev Bras Med Esporte 2006;12:405-9.

25. Perrella MM, Noriyuki PS, Rossi L. Avaliação da perda hídrica durante treino intenso de rugby. Rev Bras Med Esporte 2005;11:229-32.

26. Miguel M, Almeida D, Rossi L. Taxa de sudorese durante aula de Jump Fit e impacto da desidratação ativa no exame de BIA. Rev Ação Mov 2005; 2:74-7.

27. Petrie HJ, Stover EA, Horswill CA. Nutritional concerns for the child and adolescent competitor. Nutrition 2004;20:620-31.

28. Gorelick MH, Shaw KN, Murphy KO. Validity and reliability of clinical signs in the diagnosis of dehydration in children. Pediatrics 1997;99:E6.

29. Kenney LW, Chiu P. Influence of age on thirst and fluid intake. Med Sci Sports Exerc 2001;33:1524-32.

30. Sawka MN, Montain SJ. Fluid and electrolyte supplementation for exercise heat stress. Am J Clin Nutr 2000;72:564S-72S.

31. Greenleaf JE, Brock PJ, Keil LC, Morse JT. Drinking and water balance during exercise and heat acclimation. J Appl Physiol 1983;54:414-9.

32. Reis VAB, Azevedo COE, Rossi L. Perfil antropométrico e taxa de sudorese no futebol juvenil. Rev Bras Cineantropom Desempenho Hum 2009;11:134-41.

33. IOM (Institute of Medicine). Dietary reference intakes for water, potassium, sodium, chloride, and sulfate. Washington, DC: National Academies Press; 2004.

34. Campbell SM. Hidration needs throughout the lifespan. J Am Coll Nutr
2007;26:585S-7S

35. Maughan RJ, Leiper JB. Fluid replacement requirements in soccer. J Sports Sci 1994;12:S29-34

36. Meyer F, Bar-Or O, Wilk B. Children's perceptual responses to ingesting drinks of different compositions during and following exercise in the heat. Int J Sport Nutr 1995;5:13-24.

37. Meyer F, Bar-Or O, MacDougall D, Heigenhauser GJ. Sweat electrolyte loss during exercise in the heat: effects of gender and maturation. Med Sci Sports Exerc 1992;24:776-81.

38. Meyer F, Bar-Or O, MacDougall D, Heigenhauser GJ. Drink composition and the electrolyte balance of children exercising in the heat. Med Sci Sports Exerc 1995;27:882-7.

39. Wilk B, Kriemler S, Keller H, Bar-Or O. Consistency in preventing voluntary dehydration in boys who drink a flavored carbohydrate- $\mathrm{NaCl}$ beverage during exercise in the heat. Int J Sport Nutr 1998;8:1-9.

40. Wilk B, Bar-Or O. Effect of drink flavor and $\mathrm{NaCL}$ on voluntary drinking and hydration in boys exercising in the heat. J Appl Physiol 1996;80:1112-7.

41. Sharp RL. Role of whole foods in promoting hydration after exercise in humans. J Am Coll Nutr 2007;26 (5 Suppl):592S-6S.

42. Meyer F, Bar-Or O. Fluid and electrolyte loss during exercise. Sports Med 1994;18;4-9.

43. Saat M, Sirisinghe RG, Singh R, Tochiharan $Y$. Effects of short-term exercise in the heat on thermoregulation, blood parameters, sweat secretion and sweat composition of tropic-dwelling subjects. J Physiol Anthropol Appl Human Sci 2005;24:541-9.

44. Saat M, Tochiharan $\mathrm{Y}$, Hashigughi N, Sirisinghe RG, Fujitan M, Choun CM. Effects of Exercise in the Heat on Thermoregulation of Japanese and Malaysian Males. J Physiol Anthropol Appl Human Sci 2005;24:267-75. 